\title{
FusB energises import across the outer membrane through direct interaction with its ferredoxin substrate
}

Marta Wojnowska and Daniel Walker

Institute of Infection, Immunity and Inflammation, College of Medical, Veterinary and Life Sciences, University of Glasgow, Glasgow, G12 8QQ, UK.

Corresponding author: Daniel Walker, E-mail: daniel.walker@glasgow.ac.uk

Abbreviations: Ara - Arabidopsis, CCCP - carbonyl cyanide chlorophenylhydrazone, CTD - C-terminal domain, Fer - ferredoxin, GFP - green fluorescent protein, ITC - isothermal titration calorimetry, IUTD - intrinsically unstructured translocation domain, NTR - N-terminal region, , Pc - Pectobacterium carotovorum, PMF - proton motive force, Pot - potato, IPTG - isopropyl $\beta-D-1$ thiogalactopyranoside, SEC - size exclusion chromatography, Sp spinach, TBDT - TonB-dependent transporter. 


\begin{abstract}
Phytopathogenic Pectobacterium spp. import ferredoxin into the periplasm for proteolytic processing and iron release via the ferredoxin uptake system. Although the ferredoxin receptor FusA and the processing protease, FusC, have been identified, the mechanistic basis of ferredoxin import is poorly understood. In this work we demonstrate that protein translocation across the outer membrane is dependent on the TonB-like protein FusB. In contrast to the loss of FusC, loss of FusB or FusA abolishes ferredoxin transport to the periplasm, demonstrating that FusA and FusB work in concert to transport ferredoxin across the outer membrane. In addition to interaction with the TonB-box region of FusA, FusB also forms a complex with the ferredoxin substrate, with complex formation required for substrate transport. These data suggest that ferredoxin transport requires energy transduction from the cytoplasmic membrane via FusB for both removal of the FusA plug domain and for substrate translocation through the FusA barrel.
\end{abstract}




\section{Introduction}

Gram-negative bacteria have evolved a number of strategies for the acquisition of iron and other nutrients in which TonB-dependent transporters (TBDTs) play a central role ${ }^{1}$. In the case of siderophore-mediated iron acquisition the iron-siderophore complex is imported into the cell, captured by a siderophore-specific periplasmic binding protein and delivered to an ABC-transporter for import to the cytoplasm ${ }^{2}$. For iron acquisition from large host proteins such as transferrin, the iron-containing protein is captured at the cell surface through TBDT binding and the iron stripped at the cell surface and subsequently transported through the lumen of the $\mathrm{TBDT}^{3}$. In addition to the outer membrane receptor, whose lumen constitutes the translocation route, TBDT-mediated transport requires a complex of three proteins anchored in the inner membrane: TonB, ExbB and ExbD ${ }^{4,5}$. The ExbBD-TonB complex allows the entry of the nutrient by removal of a force-labile portion of the plug domain, which obstructs the receptor lumen ${ }^{6}$. ExbB and ExbD are related to the flagellar motor proteins and harness proton motive force to energise the transport process.

In addition to the uptake of iron siderophores and other metal chelating compounds such as vitamin $B_{12}$, TBDTs also transport complex carbohydrates and simple sugars ${ }^{7}$. A recent study has also described the role of a TonB-dependent receptor in protein export, suggesting that TonB-dependent receptors are highly adaptable to the transport of diverse substrates across the $\mathrm{OM}^{8}$. The flexibility in the range of substrates that are amenable to transport by TBDTs is exploited by protein antibiotics such as colicins and pyocins that use TBDTs as their primary cell surface receptor and translocator ${ }^{9}$. As with the uptake of nutrients, translocation of colicins and pyocins via TBDTs is PMF-dependent, although in these cases the periplasm-spanning protein TonB is required both to remove the force labile region of the TBDT-plug domain and to subsequently energise protein translocation across the $\mathrm{OM}^{10}$. Protein translocation occurs by direct interaction with the an $\mathrm{N}$-terminal intrinsically unstructured region of the toxin that, similar to the TBDTs, carries a TonB-binding motif ${ }^{10}$.

We recently demonstrated that TBDT-mediated iron acquisition from the iron-sulphur cluster containing protein ferredoxin represents an unprecedented example of protein translocation into the bacterial cell for nutrient acquision ${ }^{11}$. Ferredoxin binding at the cell surface is mediated by the TBDT FusA and, following transport of intact ferredoxin into the periplasm, the substrate is subjected to proteolytic processing by the M16 protease FusC ${ }^{11,12}$. Cleavage by Fus $C$ results in release of the iron-sulphur cluster and is required for effective iron acquisition from ferredoxin by Pectobacterium. Together with the genes encoding FusA and FusC, the Fus operon contains two additional genes, with fusB encoding a TonB-homologue and fusD a putative $A B C$ transporter ${ }^{12}$. Interestingly, the M-type pectocins M1 and M2, which we have previously described, parasitise the ferredoxin uptake 
system through an $\mathrm{N}$-terminal ferredoxin domain that is highly homologous to plant ferredoxins ${ }^{13,14}$.

More recently, the X-ray structure of FusC bound to ferredoxin has been reported, showing that substrate recognition occurs at a site distant from the active site ${ }^{15}$. Furthermore, only parts of the ferredoxin molecule are visible in the structure, implying that the bound substrate is largely unstructured. Based on these data, it was suggested that ferredoxin transport occurs by means of a Brownian ratchet mechanism in which FusC acts as a periplasmic anchor to facilitate translocation of ferredoxin across the $\mathrm{OM}$ via the lumen of Fus $\mathrm{A}^{12,15}$. Similar mechanisms have been postulated to account for mitochondrial protein uptake, whereby cytoplasmically synthesised proteins are translocated via the TOM and TIM23 complexes ${ }^{16,17}$. As such, this would represent a hitherto unexpected evolutionary link between mitochondrial and plastid protein import and bacterial protein import via the Fus and other postulated protein uptake systems.

In this work we show that FusC does not facilitate ferredoxin import and that like the import of other TBDT substrates, ferredoxin uptake is PMF-dependent. Instead, we show that the TonB-homologue encoded within the fus operon, FusB, is required for ferredoxin import and the mechanism of ferredoxin import involves a direct interaction between FusB and the ferredoxin substrate. The direct interaction of the TonB-like protein with substrate is unprecedented and explains the requirement for the system-specific TonB-homologue in the Fus system. Our data also show that, in addition to the direct interaction with the substrate, FusB fulfils another role - similar to other TonB proteins - in interacting with the TonB-box of FusA for plug displacement. Since multiple genes encoding TonB-like proteins are commonly found in the genomes of Gram-negative bacteria this may be a common mechanism for the uptake of atypical substrates via TonB-dependent receptors. 


\section{Results}

\section{Ferredoxin import is independent of FusC but requires proton motive force}

We previously showed that FusC is a highly specific protease that targets plant ferredoxin to release iron from this host protein in the periplasm of Pectobacterium spp ${ }^{11}$. However, it has also been suggested that FusC plays an additional role in iron acquisition through a direct involvement in ferredoxin transport across the outer membrane by means of a Brownian ratchet mechanism, specifically acting as a periplasmic anchor ${ }^{15}$. Our own previous work suggests that if FusC does play a role in ferredoxin import this role is not essential since the accumulation of Arabidopsis ferredoxin can still be observed in a $P$. carotovorum LMG2410 (PCLMG2410) strain lacking FusC ${ }^{11}$. However, using Arabidopsis ferredoxin $\left(\right.$ Fer $\left._{A r a}\right)$ it is not possible to directly compare the rate and extent of ferredoxin uptake between wild-type and $\triangle$ fusC $P$. carotovorum since Fer $_{\text {Ara }}$ is cleaved by FusC on import to the periplasm in the wild-type strain; hence, based on these data, we could not rule out a role for FusC in ferredoxin import.

To test the hypothesis that FusC facilitates translocation of ferredoxin to the periplasm we compared the uptake of potato ferredoxin ( Fer $_{\mathrm{Pot}}$ ) in wild-type and $\Delta f u s C$ PCLMG2410. Fer ${ }_{\text {Pot }}$ was used as we had observed that, although similarly to Fer $_{\text {Ara }}$, it can be transported into cells (Figure 1a), unlike Fer $_{\text {Ara }}$ and spinach ferredoxin $\left(\right.$ Fer $_{S p}$ ), which both support robust growth of PCLMG2410 under iron-limiting conditions ${ }^{12,13}$, it is not cleaved at an appreciable rate by FusC and so accumulates intracellularly in wild-type PCLMG2410 (Figure 1b). To compare uptake of $\mathrm{Fer}_{\text {Pot }}$ in wild-type and $\triangle$ fusC PCLMG2410, cells were grown under iron-limiting conditions through the addition of the iron chelator, 2,2'-bipyridine, and supplemented with Fer Pot. $_{\text {. The amount of Fer }}$ in in whole cell extracts and the media was determined by immunoblotting. Levels of Fer $_{\text {Pot }}$ obtained from cell extracts increased at the same rate in whole cell extracts and rates of removal of ferredoxin from the media were similar (Figure 1c). These data show that FusC does not play a role in protein import and the role of FusC in iron acquisition is likely restricted to proteolytic processing of ferredoxin as we previously reported ${ }^{11}$.

To further probe the mechanism of ferredoxin uptake we tested the ability of PCLMG2410 cells to accumulate Fer $_{\text {Pot }}$ under iron-limiting conditions and in the presence of the uncoupling agent carbonyl cyanide m-chlorophenylhydrazone (CCCP), which dissipates the PMF through transport of protons across the cytoplasmic membrane ${ }^{18}$. The intracellular accumulation of Fer $_{\text {Pot }}$ by PCLMG2410 was markedly reduced in the presence of $10 \mu \mathrm{M}$ CCCP, relative to cells grown in the absence of the uncoupling agent, and abolished at 100 $\mu \mathrm{M}$ CCCP (Figure 1d). Similar effects through the action of CCCP were observed on the intracellular accumulation of Arabidopsis ferredoxin by $\triangle$ fusC LMG2410 (Figure 1d). These data show that, analogously to other TBDT-mediated transport processes ${ }^{10,19}$, the import of 
ferredoxin is PMF-dependent and a Brownian ratchet mechanism is unlikely to play a key role in ferredoxin import in Pectobacterium spp.

\section{FusB mediates ferredoxin import into the periplasm}

As we previously reported, in addition to fusA and fusC, the Fus operon carries additional genes that encode a TonB homologue, FusB, and an $\mathrm{ABC}$ transporter FusD ${ }^{12}$. Given the documented role of TonB in siderophore import in many bacterial species we supposed FusB may play a similar role in protein import, having perhaps evolved additional functionality required to mediate the passage of a large substrate through the lumen of the TBDT FusA. To test this hypothesis we created $\triangle f u s A$ and $\triangle f u s B$ strains in PCLMG2410 and initially probed them using growth enhancement assays under iron-limiting conditions. As indicated by the loss of growth enhancement both on solid media (Figure 2a) and in liquid culture (Figure $2 b$ ), these two genes encode proteins which are essential for Fus-mediated iron acquisition. The possibility that deletion of either gene affected the expression or level of FusC, thus indirectly affecting the growth enhancement phenotype, was ruled out by immunoblotting whole cell extracts with anti-FusC antiserum (Figure S1). We further investigated the ability of the $\triangle f u s A$ and $\triangle f u s B$ PCLMG2410 to import ferredoxin relative to wild-type and $\triangle f u s C$ strains using Fer $_{\text {pot, }}$ which cannot be cleaved by FusC. Consistent with the hypothesised role of FusB in protein import, and in contrast to wild-type and $\triangle f u s C$ strains, we did not observe the intracellular accumulation of $\operatorname{Fer}_{\text {Pot }}$ in $\triangle f u s A$ and $\triangle f u s B$ PCLMG2410 (Figure 2c). The ferredoxin import phenotype lost in the $\triangle f u s A$ and $\triangle f u s B$ strains was restored by plasmid-based complementation of fus $A$ and fus $B$, respectively (Figure 2d). In these experiments the production of FusA and FusB is IPTG-inducible under the control of the T5 promoter, although in the case of FusB complementation, leaky expression in the absence of IPTG is sufficient to restore protein import.

To ensure that the abrogation of substrate import is not specific to Fer $_{\text {pot }}$ we also monitored the ability of the $\triangle f u s A$ and $\triangle f u s B$ strains to utilise Fer $_{\text {Ara. }}$. However, for this substrate instead of measuring intracellular ferredoxin accumulation, we determined loss of ferredoxin from the growth media, since accumulation of Fer $_{A r a}$ is only observed in the LMG2410 $\triangle$ fusC strain (Figure 2e). Consistent with the internalisation assay and growth enhancement assays, ferredoxin content of the media decreased over time in the presence of wild type cells, but not in the presence of the $\triangle f u s A$ and $\triangle f u s B$ strains, indicating that both FusA and FusB are required for Fer $_{\text {Ara }}$ uptake (Figure 2f).

\section{FusB directly interacts with the 'TonB-box' of FusA}


Having determined that the TonB-like protein FusB is required for ferredoxin import we aimed to elucidate the mechanism of ferredoxin uptake. Analysis of the FusA sequence showed the presence of a putative TonB-box, DTILVRST, with a similar sequence to TonBboxes from well-characterised E. coli TBDTs and other PCLMG2410 TBDTs (Figure S2). The functional importance of this putative TonB-box region was demonstrated using ferredoxin import assay and plasmid-based complementation of the $\triangle$ fusA strain, which showed that proline substitutions within the putative TonB-box abolish internalisation of the FusA substrate ferredoxin (Figure S3). The similarity of the TonB-box of FusA to the TonBboxes of other PCLMG2410 and E. coli TBDTs suggests that FusA may interact with PCLMG2410 TonB and not FusB. Although PCLMG2410 has 6 genes that encode TonB-like proteins, we hypothesised that the protein that was most similar to $E$. coli TonB, which we refer to as PCTonB, would fulfil the same function as this protein in servicing multiple TBDTs, with the primary function of dislocating the plug domain to enable substrate transport.

To determine if TonB and/or FusB interact directly with FusA we produced a construct consisting of the N-terminal region of FusA (residues 21 to 66), excluding the signal peptide region, fused to GFP (Fus $A_{N T R}-G F P$ ) and determined if this interacts with the isolated C-terminal domains of PcTonB (TonB $\mathrm{CTD}_{\mathrm{C}}$ ) and FusB (FusB $\mathrm{B}_{\mathrm{CTD}}$ ) by ITC. Clear heats of binding were observed on titration of Fus $A_{N T R}-G F P$ into FusB $B_{C T D}$, although the affinity of FusB $_{\text {CTD }}$ for Fus $A_{N T R}$-GFP is weak $(57 \mu \mathrm{M})$ (Figure 3a). No heats of binding were observed

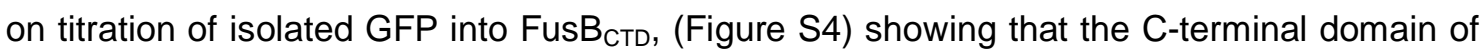
FusB specifically interacts with the $\mathrm{N}$-terminal region of FusA. Interestingly, similar heats of binding were observed on titration of FusA $\mathrm{NTR}_{\mathrm{NT}}$-GFP into TonB $\mathrm{B}_{\mathrm{CTD}}$ (Figure S5), demonstrating that the $\mathrm{N}$-terminal region of FusA can also interact with PcTonB.

To determine if the PcTonB plays a role in ferredoxin uptake, we deleted the respective gene in PCLMG2410 and tested the growth enhancement phenotype of this strain in the presence of Fer $_{\mathrm{sp}}$. In contrast to deletion of $f u s B$, the loss of tonB did not reduce growth enhancement phenotype (Figure $3 b$ ); in fact, the $\triangle$ ton $B$ strain showed more prominent zones of growth enhancement in the presence of $\mathrm{Fer}_{\mathrm{Sp}}$ relative to the wild-type strain and showed increased intracellular accumulation of Fer $_{\text {pot }}$ relative to wild-type PCLMG2410 (Figure 3c). However, PCLMG2410 $\triangle$ tonB exhibited poor growth in the presence of 2,2'-bipyridine relative to the wild-type strain (Figure 3d), with very faint growth observable after 24 hours. These data indicate that, although PcTonB does play the expected generic role in iron uptake, this does not include iron acquisition from ferredoxin. Furthermore, despite the aforementioned observation that TonB interacts with FusA in vitro (and possibly in vivo), this interaction is not sufficient for ferredoxin uptake. Indeed there may be competition between FusB and TonB for complex formation with FusA, with only the FusB-FusA complex being productive with respect to ferredoxin uptake. 


\section{FusB interacts directly with the ferredoxin substrate}

The ability of FusB and PcTonB to interact with FusA, but with only the former able to mediate ferredoxin uptake, suggests that FusB plays an additional role, which is essential for ferredoxin import. One possibility is that FusB directly interacts with the protein substrate after the initial binding of ferredoxin to FusA at the cell surface. To test this, we sought to determine if ferredoxin forms a complex with the isolated C-terminal domain of FusB by size exclusion chromatography (SEC). SEC of ferredoxin mixed with FusB $\mathrm{B}_{\text {СтD }}$ monitored at 280 $\mathrm{nm}$ and $330 \mathrm{~nm}$ gave a peak indicating the presence of a species of higher molecular weight than FusB $_{\text {CTD }}$ or Fer $_{S p}$ alone, providing evidence of complex formation (Figure 4a). In contrast, no complex formation was observed between ferredoxin and the purified C-terminal domain of TonB (TonB $\mathrm{B}_{\text {СтD }}$ ) using SEC (Figure S6). We further investigated the formation of the FusB $\mathrm{B}_{\mathrm{CTD}}-\mathrm{Fer}_{\mathrm{Sp}}$ complex by isothermal titration calorimetry, titrating $\mathrm{Fer}_{\mathrm{SP}}$ into $\mathrm{FusB}_{\mathrm{CTD}}$ (Figure 4b). These data show that FusB interacts directly with the ferredoxin substrate.

Inspection of the amino acid sequence of FusB shows that the $\mathrm{N}$-terminal portion of the predicted globular domain and the preceding linker contain a significant number of positively charged amino acids, which are absent from PCLMG2410 and E. coli TonBs (Figure S7). Two such residues (Arg176, Arg177) are found in place of the highly conserved Gln-Pro-Gln residues, which form a part of the BtuB TonB-box binding motif (QPQYP) in E. coli TonB ${ }^{20}$. This arginine motif is located within a loop/linker region of TonBs, connecting the periplasmic-spanning and globular domains. Substitution of the two FusB $\mathrm{B}_{\mathrm{CTD}}$ arginine residues with lysines rendered a folded protein that did not comigrate with ferredoxin in gel filtration or interact with the substrate in ITC experiments (Figure S8). Similarly, PCLMG2410 $\triangle$ fusB could not be complemented with a pFusB plasmid encoding the FusB R176K/R177K variant (Figure 4c). Therefore at least one of these two arginine residues appears to be critical for FusB-substrate interaction. 


\section{Discussion}

Our recent discovery that ferredoxin is imported into the periplasm of $P$. carotovorum revealed an unprecedented example of protein uptake for nutrient acquisition in Gramnegative bacteria ${ }^{11}$. In this work, we define key aspects of the mechanism of ferredoxin transport across the outer membrane. In a recent report, it was hypothesised that the M16 protease FusC acts as a periplasmic anchor that facilitates ferredoxin uptake by means of Brownian-ratchet mechanism ${ }^{15}$. However, the data presented here are inconsistent with this model, showing that ferredoxin import is independent of FusC. Instead, ferredoxin uptake requires energy transduction from the PMF and the TonB-like protein FusB. Therefore, the mechanism of ferredoxin import shares some similarity with the mechanism of import of widely studied substrates of TBDRs, such iron siderophores and vitamin $\mathrm{B}_{12}{ }^{4,21}$. For these substrates, according to the currently accepted models of TonB-dependent transport, the major role of TonB is in the displacement or partial displacement of the plug domain from their specific TBDTs ${ }^{6,10}$.

Interestingly, in the case of FusA, both FusB and PcTonB are able to interact with its $\mathrm{N}$-terminal region and so both these proteins may be able to facilitate displacement of the FusA plug domain. However, deletion of the genes encoding the two TonB proteins showed that only FusB is essential for ferredoxin transport, demonstrating an additional role for FusB in this process that cannot be fulfilled by PCTonB. Although the affinity of FusB $\mathrm{B}_{\mathrm{CTD}}$ for Fus $A_{N T R}$ is low $(57 \mu \mathrm{M})$, comparable low affinity complexes have been described between TBDT TonB-binding peptides and TonB proteins. For example, the affinity reported for the TonB-like protein HasB interaction with 21-mer HasR N-terminal peptide is $25 \mu \mathrm{M}^{22}$. Similarly weak interactions between TonB and TBDR TonB-box peptides have been reported for FhuA $(36 \mu \mathrm{M})^{23}$ and BtuB $(9.4 \mu \mathrm{M})^{6}$. However, complex formation between TonB and TonBbinding peptides is characterised by $\beta$-strand augmentation which is known to result in the formation of mechanically strong complexes ${ }^{6}$. Indeed, it has been demonstrated in vitro using atomic force microscopy that the TonB-BtuB Ton box complex is sufficiently mechanically robust to induce partial unfolding of the BtuB plug-domain, forming a channel through which the vitamin $B_{12}$ substrate can translocate ${ }^{6}$.

The ability of FusB to form a complex with ferredoxin, which PcTonB lacks, indicates that this additional role involves the direct interaction of FusB with the ferredoxin substrate and that this complex formation is essential for ferredoxin transport through the lumen of FusA. Consistent with this, we identified an arginine motif that is required for FusB-mediated ferredoxin uptake by $P$. carotovorum and formation of the FusB-ferredoxin complex. Our current model of Fus-mediated iron acquisition, whereby FusB fulfils two distinct roles, is schematically shown in Figure 5. In this model, binding of the substrate on the extracellular side of FusA releases the TonB-box into the periplasmic space, where it is captured by 
FusB. Due to the dimensions of the globular ferredoxin, which are similar to the lumen of its FusA TBDT ${ }^{11}$, ferredoxin is unlikely to be able to readily diffuse into the periplasm after removal of the FusA plug domain. We therefore hypothesise that the interaction of FusB with the substrate involves a further PMF dependent step required to pull the ferredoxin substrate through the lumen of FusA. This would involve the C-terminal domain of FusB, which is of comparable size to plant ferredoxins, to enter the lumen of FusA to contact ferredoxin on the cell surface. The FusB-ferredoxin complex can then be pulled into the periplasm, using the ExbBD complex and PMF, after which the substrate is processed by FusC. Although we present a model relying on a single FusB per import cycle, we cannot exclude the possibility that the removal of FusA plug and ferredoxin import would involve two separate FusB molecules.

The occurrence of genes encoding multiple TonB-like proteins is a common feature of many Gram-negative bacteria ${ }^{24}$ and in some cases specific TonB proteins are required for the uptake of specific substrates, as for TonB2 of Vibrio anguilarum for anguibactin uptake ${ }^{25}$, while others exhibit some level of functional redundancy ${ }^{26}$. However, to our knowledge the Fus system represents the only substrate import system in which a TonB protein has been shown to directly interact with the substrate. This additional functionality displayed by FusB may reflect the nature of the ferredoxin substrate, which is atypically large in comparison to the well-studied TBDT siderophore substrates. In this respect, the uptake of ferredoxin is similar to the TonB-dependent uptake of the colicins and pyocins, which directly interact with TonB after threading their TonB box-containing intrinsically unstructured translocation domain (IUTD) though the lumen of their respective $\mathrm{TBDT}^{10,27}$. However, since plant ferredoxins are highly stable proteins that lack any kind of similar unstructured regions, our hypothesis is that in order to contact ferredoxin, FusB must enter the FusA lumen and contact FusA-bound substrate at the cell surface. This proposed mechanism also accounts for why the ferredoxin-containing bacteriocins do not require an IUTD that contains a TonBbox to cross the $P$. carotovorum outer membrane, with FusB able to directly contact their ferredoxin receptor-binding domains at the cell surface, thus enabling parasitisation of the Fus system ${ }^{12,28}$.

In summary, we describe a novel mechanism of 'TonB-dependent' nutrient uptake that requires a direct interaction between substrate and cognate TonB protein. The occurrence of multiple TonB proteins in many Gram-negative bacteria suggests that similar mechanisms may operate for atypical TBDT substrates. 


\section{Material and Methods}

Bacterial strains and media

E. coli was grown in LB broth or plated on LB agar and grown at $37^{\circ} \mathrm{C}$. $\mathrm{DH} 5 a$ and BL21 (DE3) strains were used as host strains for cloning and for IPTG-induced protein expression, respectively. $P$. carotovorum was grown in LB broth or plated on LB agar at $30^{\circ} \mathrm{C}$ with the addition of the iron chelator 2,2'-bipyridine where specified. LB media and agar for culturing plasmid-complemented deletion strains always contained 100 $\mu \mathrm{g} \mathrm{ml}{ }^{-1}$ ampicillin.

Generation of gene knockout strains and plasmids

The fusA (KAA3668913), fusB (KAA3668912), fusC (KAA3668914) and tonB (KAA3668374) sequences were determined from the genome sequence of $P$. carotovorum LMG2410 (GenBank: BioProject PRJNA543207) ${ }^{29}$. Genes in of PCLMG2410 were deleted using the lambda red method as described previously ${ }^{11,30}$. The primers used for amplifying the kanamycin cassette from pKD4 template plasmid, gene sequences from genomic DNA and plasmid site-directed mutagenesis are listed in Table S1. The gene knockouts were confirmed by PCR and sequencing. Table S2 shows all the plasmids used in this study. To construct all plasmids, except pFusANTR-GFP, the respective genes were amplified from wild-type genomic DNA using primers that contained flanking regions with Ndel (forward) and Xhol (reverse) restriction enzyme sites. Purified PCR products were digested and ligated into $\mathrm{Ndel} / \mathrm{Xhol}$-digested pJ404, which carries ampicillin resistance. To generate pFusANTR-GFP the sequence encoding the $\mathrm{N}$-terminal portion of FusA was amplified with primers containing Xhol (forward) and BamHI (reverse) restriction enzyme sites and the PCR products were inserted into Xhol/BamHI-digested pWaldo plasmid (Waldo et al 1999). The complementation plasmids were transformed into competent LMG2410 knockout strains by electroporation.

\section{Protein production and purification}

FusC, FusB $B_{C T D}$, TonB $B_{C T D}$, Fus $A_{N T R}-G F P$ and all ferredoxin proteins were overproduced in $E$. coli and purified as described previously ${ }^{11,12}$, except spinach ferredoxin $\left(\right.$ Fer $_{\mathrm{Sp}}$ ) which was purchased from Sigma. GFP alone used as a negative control in ITC was produced by cleavage of FusA $A_{N T R}-G F P$ with TEV protease for 2 hours at RT, at 50:1 ratio. The resulting GFP-His 8 was separated from residual TEV protease by size-exclusion chromatography and the removal of the $\mathrm{N}$-terminal region of FusA was confirmed by SDS PAGE. 


\section{Growth enhancement assays}

Growth enhancement in the presence of ferredoxin was performed on solid media as previously described ${ }^{11}$. Briefly, $10 \mathrm{ml}$ of $0.8 \%$ pre-cooled agar was supplemented with $50 \mu \mathrm{l}$ of mid-log culture in LB media and poured onto an LB agar base containing $400 \mu \mathrm{M} 2,2$ 'bipyridine (and $0.2 \mathrm{mM}$ IPTG where specified). For plasmid based complementation, 100 $\mu \mathrm{g} / \mathrm{ml}$ ampicillin was added to the LB media base. $4 \mu \mathrm{l}$ of ferredoxin at specified concentration was spotted onto the solidified plate. For growth enhancement in liquid media bacteria were grown in M9 minimal media. $10 \mathrm{ml}$ cultures were inoculated with 1 in 50 dilution of overnight LB cultures and upon reaching $\mathrm{OD}_{600}=0.45$ they were supplemented with $0.2 \mu \mathrm{M}$ Fer ${ }_{A r a}$ and growth was monitored by measuring the $\mathrm{OD}_{600}$ for 6 hours.

\section{Ferredoxin internalisation and depletion assays}

The time course of ferredoxin internalisation was initiated by supplementing LB cultures of wild type or $\triangle f u s C$ cells $\left(\mathrm{OD}_{600}=0.5\right)$ with 2,2'-bipyridine to a final concentration of $200 \mu \mathrm{M}$. Fer ${ }_{\text {Ara }}$ or Fer $_{\text {Pot }}$ was added to a final concentration of $1 \mu \mathrm{M}$ and the cultures were grown at $30^{\circ} \mathrm{C}$ with shaking over the specified time. At each time point a volume equivalent to $1 \mathrm{ml}$ cell suspension at $O D_{600}=0.5$ was removed, the cells were spun down and treated with BugBuster (Merck) for soluble protein extraction. To determine if $\triangle$ fus $A$ and $\triangle f u s B$ strains can take up ferredoxin, LB cultures of WT and deletion strains at OD $\sim 0.5$ were supplemented with $200 \mu \mathrm{M}$ 2,2'-bipyridine and $1 \mu \mathrm{M}$ Arabidopsis or $5 \mu \mathrm{M}$ potato ferredoxin. After 2 hours at $30^{\circ} \mathrm{C}$ with shaking $1 \mathrm{ml}$ of cells was pelleted and soluble proteins were extracted using BugBuster (Merck). For internalisation experiments involving plasmidcomplemented deletion strains, $\mathrm{LB}$ cultures were grown until $\mathrm{OD}_{600}=0.4$ was reached, whereupon 2,2'-bipyridine and potato ferredoxin were added. $\triangle$ fus $A+p F u s A$ and $\Delta f u s B+p F u s B$ cultures were split into two separate tubes, one of which was supplemented with IPTG to a final concentration of $0.2 \mathrm{mM}$. After 2 hours cells were harvested and subjected to BugBuster extraction as described above.

Depletion of Arabidopsis ferredoxin was monitored in $2 \mathrm{ml}$ M9 minimal media cultures of WT, $\triangle$ fus $A$ and $\triangle f u s B$ strains over the course of 4 hours. Each culture, as well as $2 \mathrm{ml}$ of uninoculated media (negative control), was supplemented $100 \mu \mathrm{M}$ 2,2'-bipyridine and $1 \mu \mathrm{M}$ Fer $_{\text {Ara. }}$ At each time point $50 \mu \mathrm{l}$ of culture was removed from each tube and after pelleting the cells the supernatant was mixed with SDS loading dye.

The effect of dissipating PMF on ferredoxin uptake was determined using protonophore CCCP (Sigma), which was dissolved in DMSO to a final concentration of 10 mM. Mid-log cultures of wild type and $\triangle f$ fuSC PCLMG2410 in M9 media were supplemented with $300 \mu \mathrm{M}$ 2,2'-bipyridine and $2 \mathrm{ml}$ of each culture was mixed with $18 \mu \mathrm{l}$ DMSO and $2 \mu \mathrm{l}$ CCCP stock (for $10 \mu \mathrm{M}$ final CCCP concentration) or $20 \mu \mathrm{l} \mathrm{CCCP} \mathrm{stock} \mathrm{(10} \mu \mathrm{M}$ final CCCP 
concentration) or $20 \mu \mathrm{l}$ DMSO for the "no CCCP" control. The cultures were mixed and incubated at room temperature for $10 \mathrm{~min}$, after which wild type cultures were supplemented with $1 \mu \mathrm{M}$ Fer $_{\text {Pot }}$ and $\triangle$ fus $C$ cultures with $0.2 \mu \mathrm{M} \mathrm{Fer}_{\text {Ara. }}$. After 45 min incubation at $30^{\circ} \mathrm{C}$ with shaking, $1 \mathrm{ml}$ of each culture was pelleted, washed with $0.5 \mathrm{ml}$ PBS and subjected to BugBuster extraction.

\section{Ferredoxin cleavage assays}

Cleavage reactions were performed in $10 \mathrm{mM}$ Tris- $\mathrm{HCl}, \mathrm{pH} 7.5,50 \mathrm{mM} \mathrm{NaCl}$, with 2 $\mu \mathrm{M}$ FusC and $250 \mu \mathrm{M}$ ferredoxin at RT. At each time point $12 \mu \mathrm{l}$ were removed and mixed with SDS loading dye. Proteins were resolved on a $16 \%$ SDS PAGE gel and visualised by Coomassie staining.

\section{Analytical size-exclusion chromatography}

Proteins were concentrated to $\sim 600 \mu \mathrm{M}$ and $20 \mu \mathrm{l}$ of $T_{\text {TTD }}$ or the relevant construct of FusB $B_{\text {CTD }}$ was mixed with an equal volume of Fer $_{\mathrm{sp}}$. The mixtures were then diluted with SEC buffer (20 mM Tris- $\mathrm{HCl}, \mathrm{pH} 7.5,150 \mathrm{mM} \mathrm{NaCl}$ ) to $0.2 \mathrm{ml}$ and loaded onto Superdex 75 10/300 column (GE Healthcare), pre-equilibrated in the same buffer. Each protein was also passed through the column individually for reference. The chromatograms were recorded at both 280 and $330 \mathrm{~nm}$.

\section{Isothermal titration calorimetry}

Experiments were performed on a MicroCal $\mathrm{iTC}_{200}$ instrument (Malvern) at $25^{\circ} \mathrm{C}$ in 10 $\mathrm{mM}$ Tris $-\mathrm{HCl} \mathrm{pH} 7.5,150 \mathrm{mM} \mathrm{NaCl}$, with differential power set to 3 . All proteins were dialysed against the ITC buffer overnight at $4^{\circ} \mathrm{C}$ except Fus $_{\text {CTD }}$ and $T{ } B_{C T D}$, which were passed through gel filtration in ITC buffer immediately before the experiment. Each type of titration was repeated at least once using different batches of purified proteins. $2 \mu$ injections were used and titrations were continued until the signal represented heats of dilution. The magnitude of heats of dilution for each titrant was established in a separate experiment, where the titrant was injected into buffer. $K_{d}$ values are expressed as the mean ( $\left.\pm S E M\right)$. 


\section{References}

1. Krewulak, K. D. \& Vogel, H. J. TonB or not TonB: is that the question? Biochem. Cell Biol. 89, 87-97 (2011).

2. Noinaj, N., Guillier, M., Barnard, T. J. \& Buchanan, S. K. TonB-dependent transporters: regulation, structure, and function. Annu. Rev. Microbiol. 64, 43-60 (2010).

3. Noinaj, N. et al. Structural basis for iron piracy by pathogenic Neisseria. Nature $\mathbf{4 8 3}$, 53-58 (2012).

4. Celia, H. et al. Structural insight into the role of the Ton complex in energy transduction. Nature 538, 60-65 (2016).

5. Maki-Yonekura, S. et al. Hexameric and pentameric complexes of the ExbBD energizer in the Ton system. Elife 7, (2018).

6. Hickman, S. J., Cooper, R. E. M., Bellucci, L., Paci, E. \& Brockwell, D. J. Gating of TonB-dependent transporters by substrate-specific forced remodelling. Nat. Commun. 8, 14804 (2017).

7. Glenwright, A. J. et al. Structural basis for nutrient acquisition by dominant members of the human gut microbiota. Nature 541, 407-411 (2017).

8. Gómez-Santos, N., Glatter, T., Koebnik, R., Świątek-Połatyńska, M. A. \& SøgaardAndersen, L. A TonB-dependent transporter is required for secretion of protease PopC across the bacterial outer membrane. Nat. Commun. 10, 1360 (2019).

9. Kleanthous, C. Swimming against the tide: progress and challenges in our understanding of colicin translocation. Nat. Rev. Microbiol. 8, 843-848 (2010).

10. White, P. et al. Exploitation of an iron transporter for bacterial protein antibiotic import. Proc. Natl. Acad. Sci. 114, 12051-12056 (2017).

11. Mosbahi, K., Wojnowska, M., Albalat, A. \& Walker, D. Bacterial iron acquisition mediated by outer membrane translocation and cleavage of a host protein. Proc. Natl. Acad. Sci. U. S. A. 115, 6840-6845 (2018).

12. Grinter, R. et al. Structure of the bacterial plant-ferredoxin receptor FusA. Nat. Commun. 7, (2016).

13. Grinter, R., Milner, J. \& Walker, D. Ferredoxin Containing Bacteriocins Suggest a Novel Mechanism of Iron Uptake in Pectobacterium spp. PLoS One 7, e33033 (2012). 
14. Grinter, R. et al. Structure of the atypical bacteriocin pectocin M2 implies a novel mechanism of protein uptake. Mol. Microbiol. 93, 234-46 (2014).

15. Grinter, R. et al. FusC, a member of the M16 protease family acquired by bacteria for iron piracy against plants. PLOS Biol. 16, e2006026 (2018).

16. Neupert, W. \& Brunner, M. The protein import motor of mitochondria. Nat. Rev. Mol. Cell Biol. 3, 555-565 (2002).

17. Backes, S. \& Herrmann, J. M. Protein Translocation into the Intermembrane Space and Matrix of Mitochondria: Mechanisms and Driving Forces. Front. Mol. Biosci. 4, 83 (2017).

18. Kasianowicz, J., Benz, R. \& McLaughlin, S. The kinetic mechanism by which CCCP (carbonyl cyanidem-Chlorophenylhydrazone) transports protons across membranes. J. Membr. Biol. 82, 179-190 (1984).

19. Braud, A., Hannauer, M., Mislin, G. L. A. \& Schalk, I. J. The Pseudomonas aeruginosa pyochelin-iron uptake pathway and its metal specificity. J. Bacteriol. 191, 3517-25 (2009).

20. Shultis, D. D., Purdy, M. D., Banchs, C. N. \& Wiener, M. C. Outer membrane active transport: structure of the BtuB:TonB complex. Science 312, 1396-9 (2006).

21. Ferguson, A. D. et al. Structural basis of gating by the outer membrane transporter FecA. Science 295, 1715-9 (2002).

22. Amorim, G. C. de et al. The Structure of HasB Reveals a New Class of TonB Protein Fold. PLoS One 8, e58964 (2013).

23. Sean Peacock, R., Weljie, A. M., Peter Howard, S., Price, F. D. \& Vogel, H. J. The solution structure of the $\mathrm{C}$-terminal domain of TonB and interaction studies with TonB box peptides. J. Mol. Biol. 345, 1185-1197 (2005).

24. Schauer, K., Rodionov, D. A. \& de Reuse, H. New substrates for TonB-dependent transport: do we only see the 'tip of the iceberg'? Trends Biochem. Sci. 33, 330-8 (2008).

25. López, C. S., Peacock, R. S., Crosa, J. H. \& Vogel, H. J. Molecular characterization of the TonB2 protein from the fish pathogen Vibrio anguillarum. Biochem. J. 418, 49-59 (2009).

26. Paquelin, A., Ghigo, J. M., Bertin, S. \& Wandersman, C. Characterization of HasB, a Serratia marcescens TonB-like protein specifically involved in the haemophore- 
dependent haem acquisition system. Mol. Microbiol. 42, 995-1005 (2001).

27. Cascales, E. et al. Colicin Biology. Microbiol. Mol. Biol. Rev. 71, 158-229 (2007).

28. Grinter, R. et al. Structure of the atypical bacteriocin pectocin M2 implies a novel mechanism of protein uptake. Mol. Microbiol. 93, (2014).

29. Rooney, W. M., Wojnowska, M. \& Walker, D. Draft Genome Sequence of the Necrotrophic Plant-Pathogenic Bacterium Pectobacterium carotovorum subsp. carotovorum Strain LMG 2410. Microbiol. Resour. Announc. 8, e00614-19 (2019).

30. Datsenko, K. A. \& Wanner, B. L. One-step inactivation of chromosomal genes in Escherichia coli K-12 using PCR products. Proc. Natl. Acad. Sci. 97, 6640-6645 (2000). 


\section{Figure Legends}

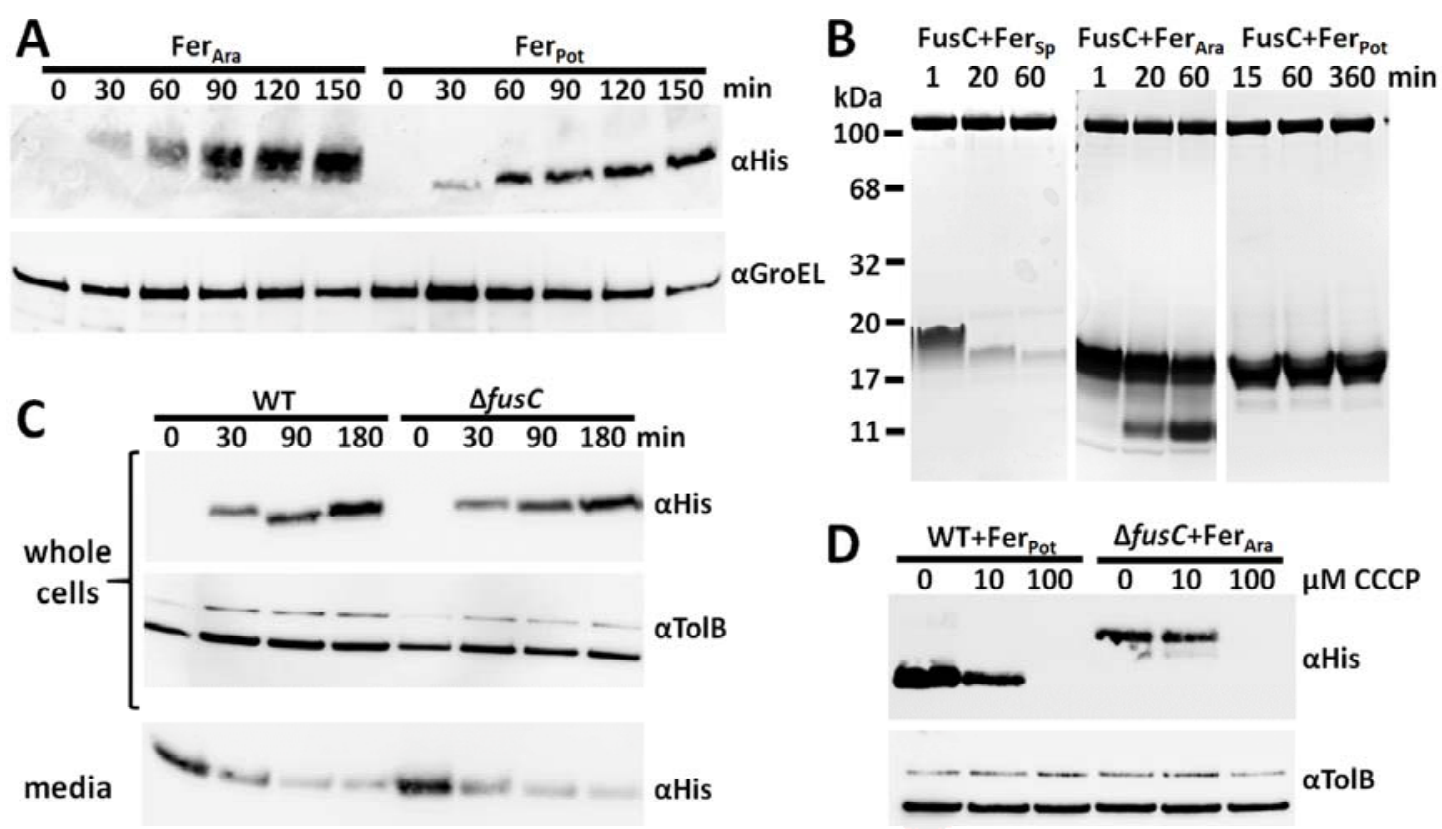

Figure 1. Import of ferredoxin is FusC-independent and requires proton motive force.

(A) Uptake of Fer $_{\text {Ara }}$ and Fer Pot $_{\text {by }} \triangle$ fusC cells over time determined by immunoblotting of whole cell extracts; GroEL serves as loading control. (B) FusC-mediated cleavage assays of plant ferredoxins. (C) Comparison of Fer $_{\text {pot }}$ uptake by wild-type (WT) and $\Delta$ fusC cells with TolB as the loading control; the bottom panel showing the concomitant depletion of ferredoxin from the media. (D) Ferredoxin import assays in the presence of protonophore CCCP; Fer ${ }_{\text {Pot }}$ was used as a reporter in wild type cells while Fer $_{\text {Ara }}$ was used in $\triangle$ fusC cells. 


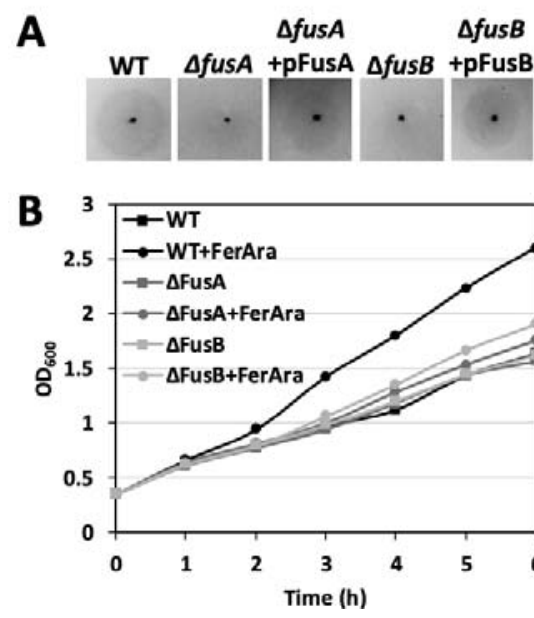

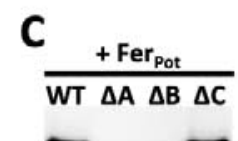
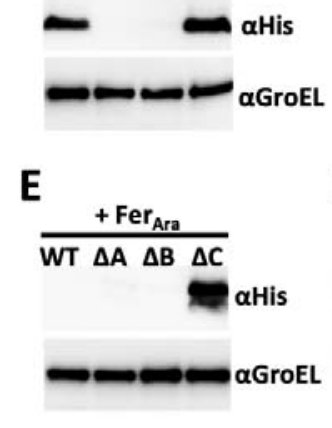

D

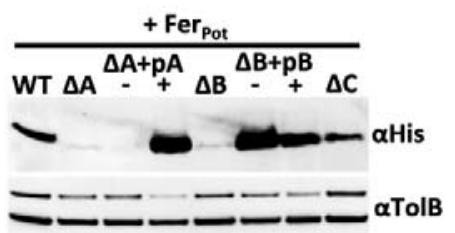

$\mathbf{F}$
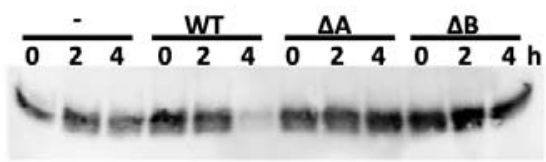

Figure 2. Fus $A$ and FusB are required for ferredoxin uptake. (A) Growth enhancement assay using spinach ferredoxin spotted onto soft agar overlay containing wild-type (WT), deletion strains and deletion strains recomplemented with the respective plasmid. (B) Growth curve comparing the rate of growth of wild-type and each deletion strain in the absence and presence of Arabidopsis ferredoxin. (D) Internalisation assay of Fer Pot $_{\text {; mid-log }}$ phase cells of each strain were supplemented with 2,2'-bipyridine and $1 \mu \mathrm{M}$ ferredoxin, grown for 1 hour and whole cell extracts were probed with anti-His antiserum. (E) Internalisation assay of Fer $_{\text {Pot }}$ including deletion strains recomplemented in trans using plasmids encoding the respective genes (" $\Delta A+p A "=\Delta f u s A+p F u s A, " \Delta B+p B "=$ $\Delta$ fusB+pFusB); prior to the addition of 2,2'-bipyridine and ferredoxin, the cultures of recomplemented strains were split in two and either supplemented with 0.5mM IPTG ("+") or grown in the absence of inducer ("-"). (F) Internalisation assay using Fer $_{\text {Ara }}$ (see D). (G) Depletion assay showing the gradual reduction of Arabidopsis ferredoxin level in the media derived from uninoculated (-), wild-type, $\Delta f u s A(\Delta \mathrm{A})$ and $\Delta f u s B(\Delta \mathrm{B})$ cell cultures. 

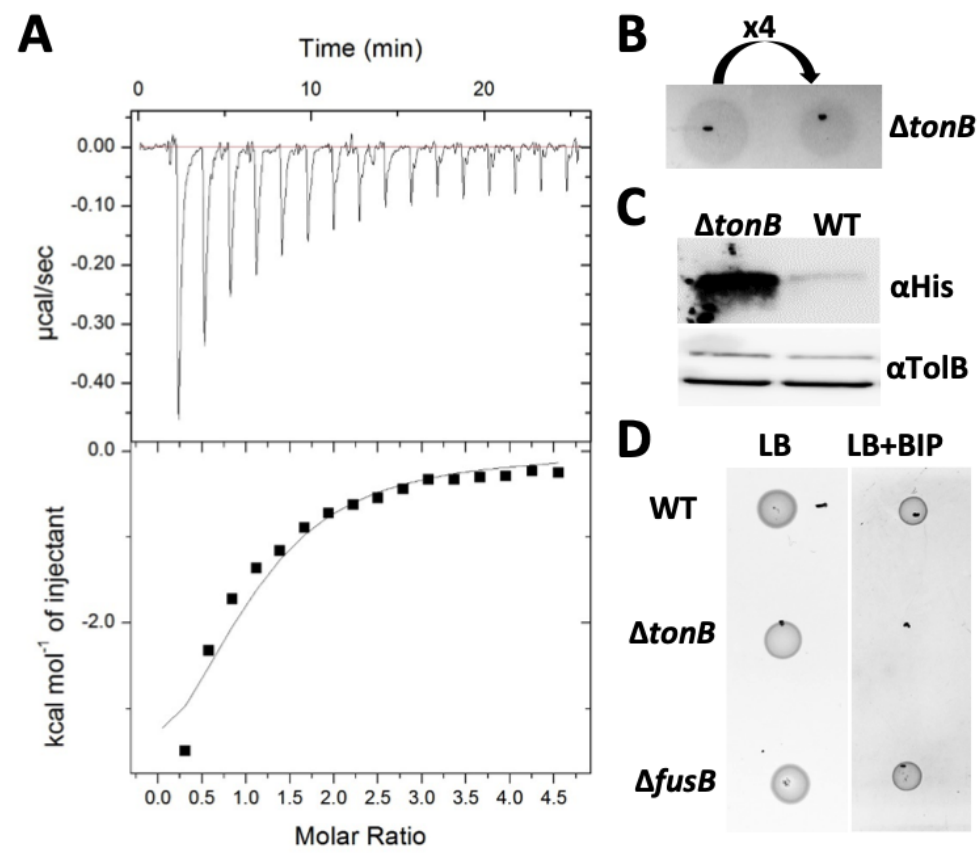

Figure 3. FusB interacts with the Fus $A$ 'TonB-box' and ExbB, but not PcTonB, is required for ferredoxin uptake. (A) ITC binding isotherm of $1 \mathrm{mM}$ Fus $A_{N T R}-G F P$ titrated into $90 \mu \mathrm{M}$ FusB $B_{C T D}$. The calculated $K_{d}$ for the Fus $A_{N T R}$-GFP-FusB $B_{C T D}$ complex is $57( \pm 12) \mu \mathrm{M}$ $(n=3)$. (B) Growth enhancement assay using spinach ferredoxin on soft agar overlay containing $\triangle$ tonB cells; $x 4$ refers to dilution factor. (C) Uptake of potato ferredoxin by $\Delta$ ton $B$ and wild-type cells over the course of 1 hour. (D) Growth assay of wild type (WT), $\Delta$ tonB and $\triangle$ fusB cells spotted onto LB agar (LB) or LB agar supplemented with $400 \mu \mathrm{M} 2,2$ '-bipyridine $(\mathrm{LB}+\mathrm{BIP})$. 

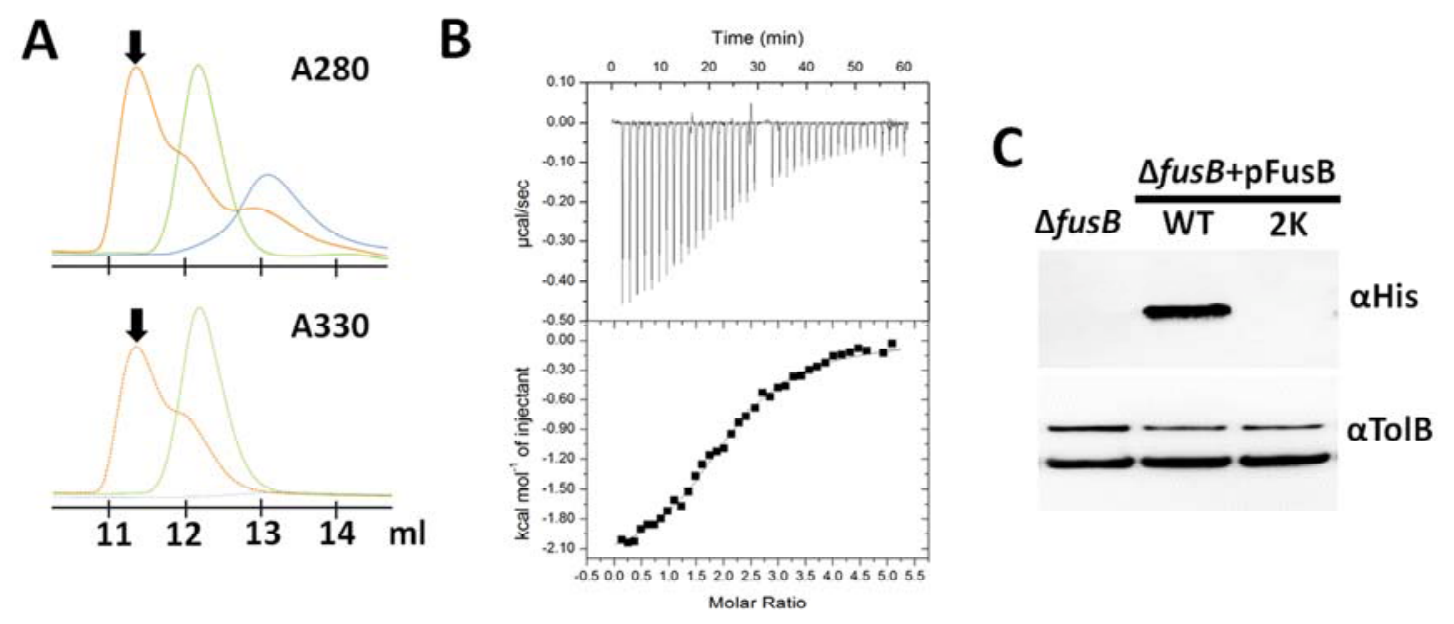

Figure 4. FusB interacts with ferredoxin substrate. (A) Overlaid size exclusion chromatograms of FusB $_{C T D}$ (blue), spinach ferredoxin (green) and a mixture of the two proteins (orange) with the arrow pointing at the ferredoxin-FusB $\mathrm{B}_{\text {СтD }}$ complex. (B) ITC binding isotherm of $600 \mu \mathrm{M}$ Fer $_{S p}$ titrated into $70 \mu \mathrm{M}$ FusB $_{\text {CTD }}$. The calculated $K_{d}$ for the Fer $_{S_{p}}$ FusB $_{\text {CTD }}$ complex is $8.7( \pm 3.5) \mu \mathrm{M}(\mathrm{n}=3)$. (C) Ferredoxin import assay showing the level of potato ferredoxin uptake by $\triangle f u s B$ cells complemented with plasmids encoding either wild type (WT) or FusB CTD R176K/R177K (2K). 


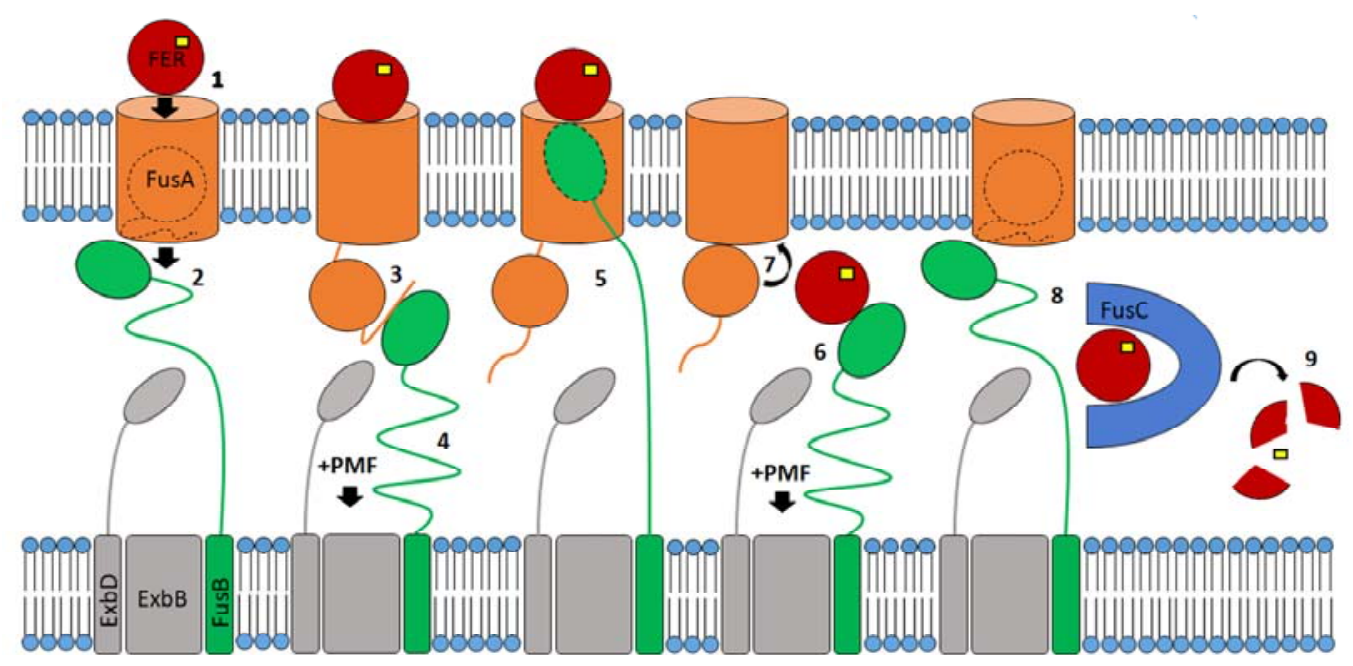

Figure 5. Proposed mechanism of FUS-mediated ferredoxin import mechanism. In the proposed mechanism FusB (green) fulfils two roles, firstly in displacement of the FusA plug domain and secondly in directly mediating ferredoxin translocation via the FusA lumen. Binding of ferredoxin (red) to FusA at the cell surface (1) causes release of the FusA TonBbox into the periplasm (2) where it is bound by FusB, which dislocates the plug domain (3) through energy transduced from the PMF via the ExbBD complex (4). FusB is then able to enter the lumen of FusA, bind ferredoxin (5) and through transduction of the PMF, translocate ferredoxin into the periplasm (6). The plug domain is then able to re-enter the FusA barrel (7) and return the FusA and FusB proteins to their resting states. Ferredoxin is then bound by FusC in the periplasm (8), which proteolytically cleaves the substrate releasing the iron-sulphur cluster (yellow) (9). 


\section{Supplementary Information}

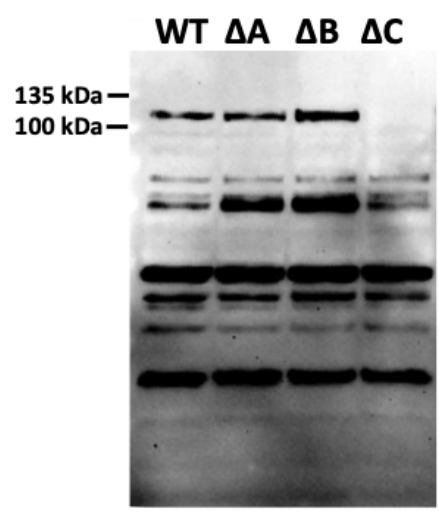

Figure S1. FusC is produced in $\Delta f u s A$ and $\Delta f u s B$ strains. Immunoblot showing the level of FusC $(101 \mathrm{kDa})$ in the periplasmic fractions extracted from wild-type, $\Delta f u s A(\Delta \mathrm{A}), \Delta f u s B$ $(\triangle \mathrm{B})$ and $\triangle$ fus $C(\Delta \mathrm{C})$ cells grown to mid-log phase in the presence of 2,2'-bipyridine.

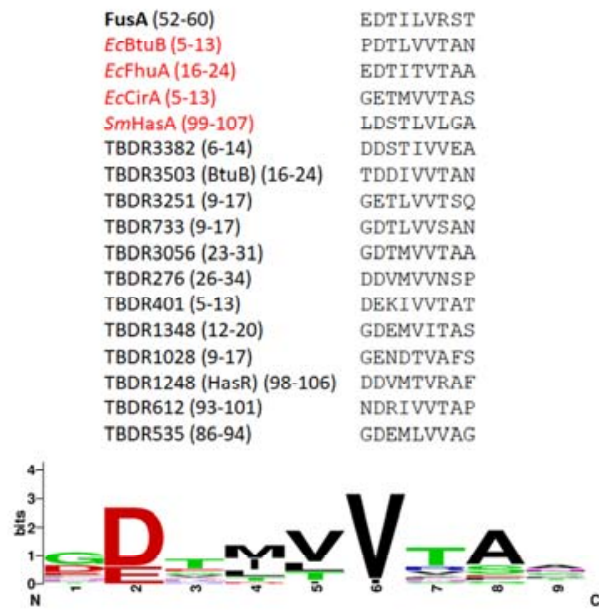

Figure S2. Sequence alignment of the putative TonB box regions of FusA against other Pc LMG2410 and E. coli TonB box regions. The numbers refer to the position of the predicted TonB box in the mature protein. Three Pc LMG2410 proteins were not included as their TonB boxes were not clearly identifiable. Sm refers to Serratia marcescens, Ec to $E$. coli. The consensus sequence shown below was generated using Weblogo. 


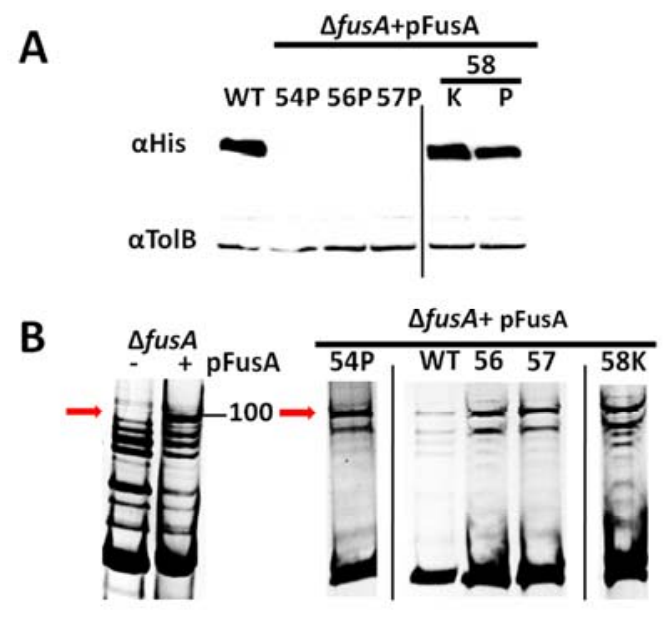

Figure S3. Mutations within the putative Fus A TonB box affect ferredoxin transport. A - potato ferredoxin import assay of $\triangle$ fus $A$ cells recomplemented with gene encoding either wild type or FusA variants. B - outer membrane extracts confirming the expression and appropriate localisation of wild type and mutant FusA.
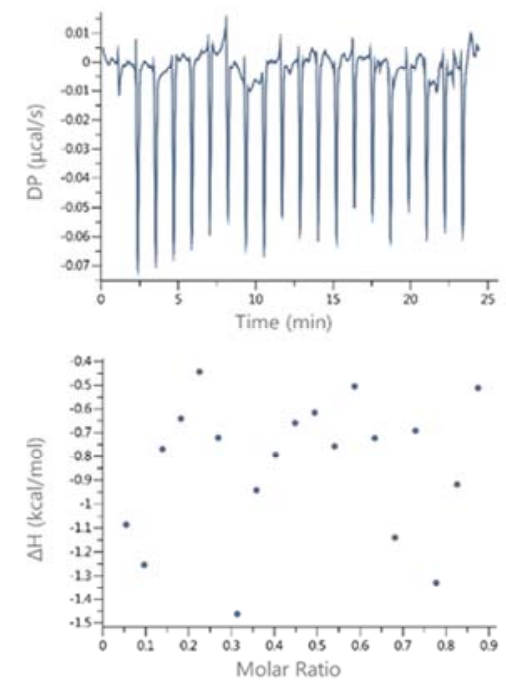

Figure S4. GFP does not interact with FusB $B_{\text {ctD. }}$ Titration of $1.2 \mathrm{mM} \mathrm{GFP}$, resulting from TEV mediated cleavage of Fus $A_{N T R}-G F P$, into $60 \mu \mathrm{M}$ Fus $B_{C T D}$. 


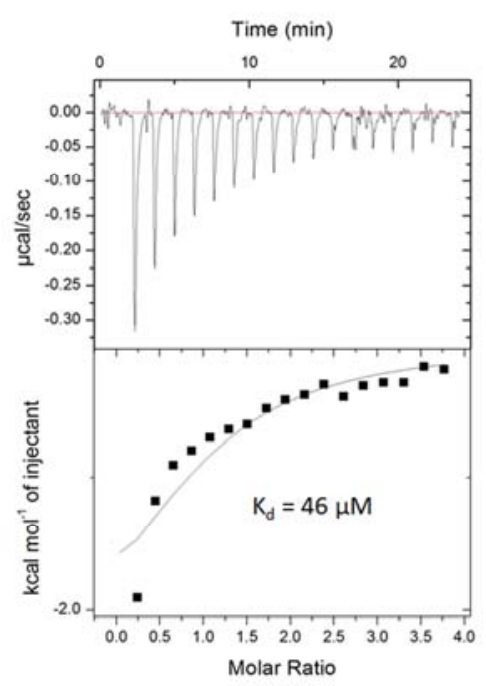

Figure S5. TonB $\mathrm{B}_{\text {Ст }}$ forms a complex with the $\mathrm{N}$-terminal domain of FusA. ITC was performed by titrating $1.2 \mathrm{mM}$ Fus $_{\mathrm{NTR}}$-GFP into $45 \mu \mathrm{M}$ TonB $\mathrm{CTD}_{\text {. }}$.
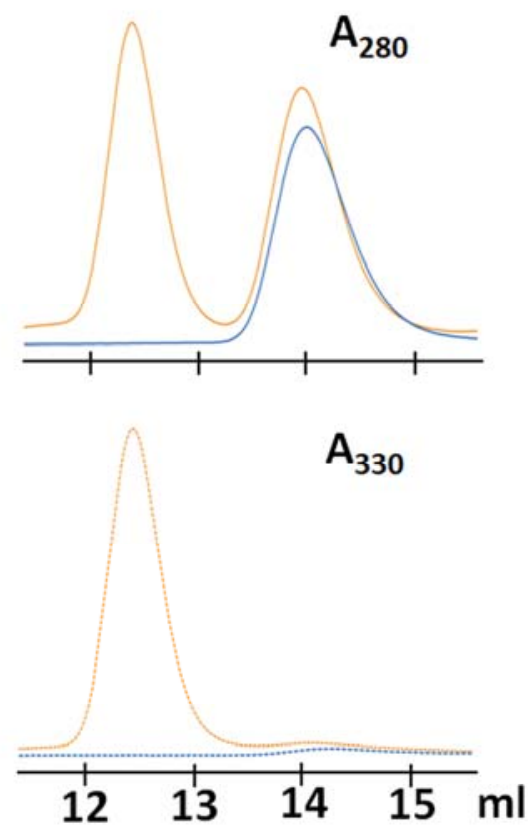

Figure S6. TonB $\mathrm{B}_{\text {CTD }}$ does not form a complex with ferredoxin. Overlay of size-exclusion chromatograms showing $\mathrm{TonB}_{\mathrm{CTD}}$ in the absence (blue trace) and in the presence of Fer $_{\mathrm{SP}}$ (orange trace). 


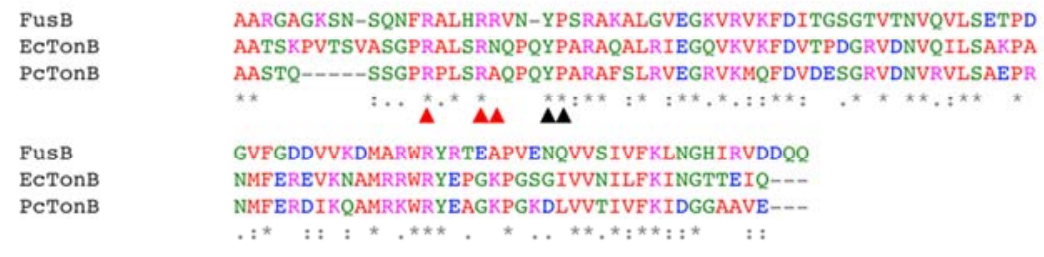

Figure S7. ClustalW sequence alignment of the C-terminal domains of FusB, PcTonB and EcTonB. Alignments start at residue 211 of FusB, 158 of PcTonB and 140 of EcTonB. Black arrows indicate the highly conserved Tyr and Pro residues, red ones show arginines in FusB that are present in the first loop of the $\mathrm{C}$-terminal domain.
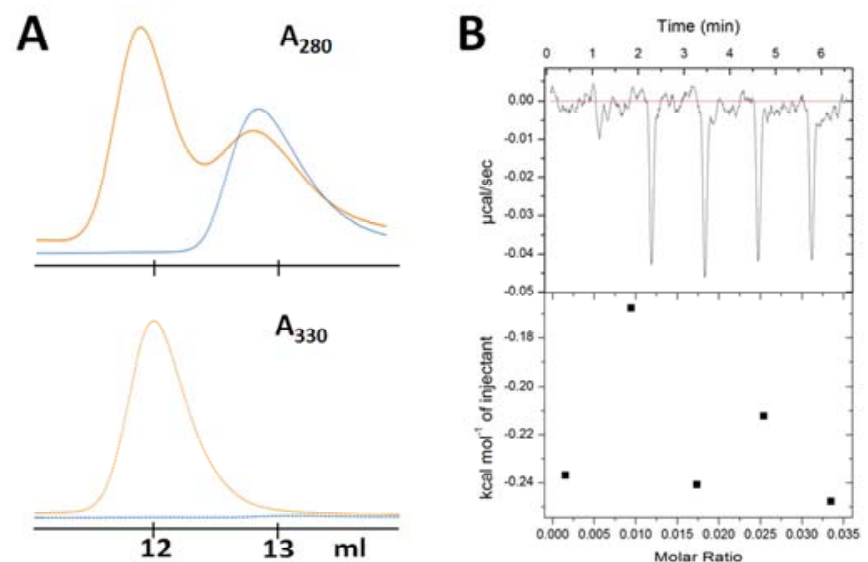

Figure S8. Substitution of two arginine residues in FusB ${ }_{\text {СтD }}$ with two lysines precludes interaction with ferredoxin molecule. A - size exclusion chromatograms at 280 and $330 \mathrm{~nm}$ of $\mathrm{FusB}_{\mathrm{CTD}} 2 \mathrm{~K}$ alone (blue) or in the presence of spinach ferredoxin (orange) showing no complex formation. B - ITC titration of $\mathrm{Fer}_{\mathrm{Sp}}$ into $\mathrm{FusB}_{\mathrm{CTD}} 2 \mathrm{~K}$ showing only heats of dilution. 
Table S1. List of primers used in this study.

\begin{tabular}{|c|c|c|}
\hline $\begin{array}{c}\text { Target } \\
\text { plasmid/ } \\
\text { gene }\end{array}$ & Primer name & Primer sequence $\left(5^{\prime} \rightarrow 3^{\prime}\right)$ \\
\hline \multirow[t]{2}{*}{ fusA knockout } & FusAKOfor & $\begin{array}{l}\text { CACATGGAATTTACAATAATATTTTATTTTTAAAATGAT } \\
\text { TATGCGTGTAGGCTGGAGCTGCTTC }\end{array}$ \\
\hline & FusAKOrev & $\begin{array}{l}\text { GGATTTACCAGGTGTAAGCGACGCCAAGCCAGAACT } \\
\text { GACGGCCCATATGAATATCCTCCTTAG }\end{array}$ \\
\hline \multirow[t]{2}{*}{ fusB knockout } & FusBKOfor & $\begin{array}{l}\text { CTGCACTACTGACACCAGGAAGTTGAAGTTTAAGAG } \\
\text { ATGGAGTTGGGTGTAGGCTGGAGCTGCTTC }\end{array}$ \\
\hline & FusBKOrev & $\begin{array}{l}\text { CCCAATAAGCATAATCATTTTAAAAATAAAATATTATT } \\
\text { GTAAATTCCCATATGAATATCCTCCTTAG }\end{array}$ \\
\hline \multirow[t]{2}{*}{ TonB knockout } & TonBKOfor & $\begin{array}{l}\text { CGTGATGTTGTTTGAAGACAATAGTGCGAGGACAATA } \\
\text { GGTTGGTGTAGGCTGGAGCTGCTTC }\end{array}$ \\
\hline & TonBKOrev & $\begin{array}{l}\text { CGCGCTCGAACATATTGCGTGGCTCAGCAGAAAGCA } \\
\text { CGCGCACGCATATGAATATCCTCCTTAG }\end{array}$ \\
\hline \multirow[t]{2}{*}{ ExbB knockout } & ExbBKOfor & $\begin{array}{l}\text { GTACAGAACAGATGATTGTACAGAACGAATGGTGGA } \\
\text { GGCACAGCGTGTAGGCTGGAGCTGCTTC }\end{array}$ \\
\hline & ExbBKOrev & $\begin{array}{l}\text { CGTTGCTGGCAGCCACATCGAGATCGCGACTTTGCA } \\
\text { GCAGCAGCATATGAATATCCTCCTTAG }\end{array}$ \\
\hline \multirow[t]{2}{*}{ pFusA } & FusAfor & GGAATTCCATATGAATAAGAACGTCTATTTAATG \\
\hline & FusA rev & CAGCTCGAGTTACCAGGTGTAAGCGAC \\
\hline \multirow[t]{2}{*}{ pFusB } & FusBfor & GGAATTCCATATGAGTAGTGAAAATCTTC \\
\hline & FusBrev & CTGCTCGAGTTATTGCTGGTCATCAAC \\
\hline \multirow[t]{2}{*}{ pFusB-CTD } & FusBCTDfor & GGAATTCCATATGGCGGCAACGGCCAAGG \\
\hline & FusBCTDrev & CACCTCGAGTTGCTGGTCATCAAC \\
\hline \multirow[t]{2}{*}{ pTonB-CTD } & TonBCTDfor & GGAATTCCATATGGCAGCAAGCACGCAGTC \\
\hline & TonBCTDrev & CACCTCGAGTTCGACTGCCGCCCCGCC \\
\hline \multirow[t]{2}{*}{ pExbB } & ExbBfor & GGAATTCCATATGAAGACGGCTGTCAGTAATAC \\
\hline & ExbBrev & CACCTCGAGTTAACCCACCCGCAGTTTATG \\
\hline \multirow[t]{2}{*}{$\begin{array}{l}\text { pFusANTR- } \\
\text { GFP }\end{array}$} & FusANTRfor & GAGCTCGAGATGCAGCAAAATGATACCTCTG \\
\hline & FusANTRrev & CACGGATCCCATCGACTGACTAGTCGGGG \\
\hline \multirow[t]{2}{*}{ pFusA-E49P } & FusAE49Pfor & GTGATTCCTCTTCGCCGAACGGCGAAGATAC \\
\hline & FusAE49Prev & GTATCTTCGCCGTTCGGCGAAGAGGAATCAC \\
\hline
\end{tabular}




\begin{tabular}{|l|l|l|}
\hline pFusA-E52P & FusAE52Pfor & CTTCGGAAAACGGCCCGGATACGATTTTAGTC \\
\hline & FusAE52Prev & GACTAAAATCGTATCCGGGCCGTTTTCCGAAG \\
\hline
\end{tabular}

Table S2. List of plasmids used in this study

\begin{tabular}{|l|l|l|}
\hline \multicolumn{1}{|c|}{ Plasmid name } & \multicolumn{1}{c|}{ Vector backbone } & \multicolumn{1}{c|}{ Source } \\
\hline pKD4 & pANTSY & Datsenko \& Wanner 2000 \\
\hline pKD46 & pINT-ts & Datsenko \& Wanner 2000 \\
\hline FusA ${ }_{\text {NTR }}$-GFP & pWaldo (Waldo et al 1999) & This study \\
\hline pFusA & pJ404 & This study \\
\hline pFusB & pJ404 & This study \\
\hline pFusC & pJ404 & Mosbahi et al 2018 \\
\hline pFusB-CTD & pJ404 & This study \\
\hline pTonB-CTD & pJ404 & This study \\
\hline pExbB & pJ404 & This study \\
\hline pFerAra & pET21 & Grinter et al 2016 \\
\hline pFerPot & pET21 & Grinter et al 2016 \\
\hline
\end{tabular}

